\title{
REVALORIZACIÓN DE LA FORMA EN LA PLANIFICACIÓN DE LOS TERRITORIOS URUGUAYOS: mensajes de la geografía y de la herencia patrimonial
}

\author{
Pablo Alfredo Ligrone \\ Dr. Arquitecto por la Sorbona Nouvelle/Paris 3, Francia \\ Profesor de la Universidad de la República/Uruguay
}

\section{RESUMEN}

Hay momentos en la vida de una ciudad o de un territorio que demandan una nueva forma que reoriente su evolución, que fije, que innove, que dé imagen de marca, que relance otro ciclo. Una nueva forma, descubierta o inventada, un motivo de diseño que oriente su transformación física, la de su geografía, su dimensión espacial.

Este trabajo muestra cómo en los últimos 15 años varios documentos de planificación en Uruguay han buscado poner en valor los mensajes de la geografía y de la herencia patrimonial a través de la forma a diferentes escalas territoriales: los arcos costeros, el relieve, las cuencas, los trazados fundacionales, sistemas de componentes patrimoniales, paisajes caracterizados, sistemas de espacios públicos, espacios de integración y lugares con su genio propio. 
ID_INVESTIGACIONES

Reivindica el valor del proyecto urbano y del proyecto de territorio y de la dimensión formal asociada al paisaje natural y cultural.

Palabras clave: paisajes culturales, forma urbana, planificación, Uruguay.

\section{ABSTRACT}

There are moments in the life of a city or a territory, which demand the apparition of a new shape to reorient their evolution, to ratify and innovate, to give a brand image, to launch a new cycle. A new discovered or invented shape, a design motif orientating its physical and geographical transformation, its spatial dimension.

This article illustrates how in the last 15 years several planning documents in Uruguay have sought to revaluate, through their shape, the messages of geography and patrimonial heritage, at different territorial scales: coastal arches, landform, watersheds, foundational outlines, heritage-component systems, characterized landscapes, public-space systems, spaces for integration and places with their own genius.

It claims for the value of the urban and territorial project and the shape associated with the natural and cultural landscape.

Key words: cultural landscapes, urban shape, planning, Uruguay 


\section{ID I NVESTIG AC I O NES}

\section{INTRODUCCIÓN}

La falsa disyuntiva plan-proyecto de la que Giuseppe Campos Venuti daba cuenta a comienzos de los 80 , al igual que los innumerables análisis críticos sobre las virtudes y limitaciones de la planificación en sus múltiples escalas, mantienen viva, hasta el presente, la dificultad de integrar los desafíos que plantean la orientación de los procesos territoriales y la conformación y estructuración del territorio resultante.

Por una parte, los enfoques sistémicos dan cuenta de complejidades y redes; por otra, el manejo de la forma y de la estructura permite analizar y actuar en la conformación de tejidos urbanos y rurales, naturales y productivos, espacios públicos y equipamientos.

En momentos de concebir los instrumentos de planificación, las capacidades disciplinares con que cuentan los gobiernos de los territorios -cantones, municipios, ciudades, áreas metropolitanas, regiones- enfrentan el reto, no siempre consciente, del manejo solvente, inteligente y sensible de sistemas y formas. En ésto no existe contradicción, existe dificultad.

Muchas de estas formas están latentes, como las infinitas figuras que logra extraer el escultor de un trozo de mármol en bruto. El arquitecto y sus atentos sentidos han de entrenarse para esta tarea. No importa la escala o complejidad de la porción de territorio a trabajar. Las formas, las piezas, las relaciones están allí: hace falta descubrirlas, ponerlas en evidencia y hacerlas trabajar unas con otras.

Hay ciudades que han tenido un plano inicial concebido con gestos fuertes, esquemas estructurantes e ideas fuerza que le dieron su personalidad durante décadas o centurias. Otras, nacidas de la simple sumatoria de construcciones, han adquirido una forma aleatoria - con trazados sinuosos, intrincados, lineales, más o menos orgánicos- logrando altas calidades urbanas. Pero también las 


\section{ID_INVESTIG AC I O NES}

hay, y demasiadas, que nacen de simples loteos sin pensamiento reflexivo, sin ideas ni esmero alguno, o urbanizaciones que crecieron por repetición anodina, con apariencia "infinita", de suburbios de vivienda individual o de bloques colectivos. En las últimas décadas, en el Cono Sur americano, ha sido común la creación de loteos en cuyos trazados no se aprecian otras "preocupaciones" que las indispensables para resolver alguna necesidad fundacional de afincar población errante, de especulación inmobiliaria, de crear soportes para la habitación en fronteras de expansión agrícola, la solución masificada de habitación social, etc.

En cualquier caso, las ciudades, con plan o sin él, han sufrido períodos, a veces demasiado prolongados, de expansión sin reflexión, producto del laissez faire, laissez passer que repite soluciones burocráticas, sin identidad, sin alma.

Este trabajo pretende llamar la atención sobre la relevancia del diseño creativo en el proyecto de ciudades y de territorios específicos, aplicado a momentos fundamentales como lo deberían ser los de planificación. También lo hace sobre la importancia que la mirada atenta del proyectista tiene, respecto de los sistemas de espacios públicos, de la morfología urbana, de la arquitectura de la ciudad y del modelado del paisaje. ${ }^{1}$ Focaliza entonces sobre una de las responsabilidades disciplinares irrenunciables del arquitecto: el manejo de la forma, del espacio y de la estética.

${ }^{1}$ Quiero destacar una obra sobresaliente de un investigador portugués, arquitecto sensible, docente y excelente dibujante: RESSANO GARCIA LAMAS, José Manuel (1993) Morfología e desenho de Cidade. Lisboa, Fundaçao Calouste Gulbenkian. Segunda edición 1993, basada en su tesis de doctorado, 1989. 


\section{ID_INVESTIGAC I ONES}

\section{RECUPERAR LA DIMENSIÓN FORMAL}

El ausentismo creciente de la forma como dimensión sustantiva en la planificación y en el proyecto urbano tiene varias causas: la depreciación de la estética, el embate de las ciencias sociales, la posición de marginalidad del sistema de espacios públicos, los excesos del formalismo y del funcionalismo, y la involución disciplinar de la arquitectura y del urbanismo.

Demasiado se ha denostado a la estética y a la belleza en las escuelas de arquitectura y en los posgrados de urbanismo por ser "políticamente incorrecto" hablar de ellas o no estar "a la moda"; demasiada desproporción en favor de la gestión, la planificación, la cantidad, la eficiencia -aunque tal grado de preocupación no repercuta en una proporcional efectividad- en desmedro de la calidad del diseño y de la ejecución del espacio público. Esto ha llevado a dejar de utilizar en la formación profesional buena parte de la bibliografía referida al espacio perceptivo, espacio existencial, espacio cultural, antropología del espacio, semiología, entre otras. Resultado: la copia banal de referentes comercialmente exitosos ha sustituido el estudio sobre las bases y principios del diseño.

Las tendencias que dieron entrada triunfal a la sociología, la economía y la política en la cuestión urbana, en lugar de enriquecer el análisis sistémico de las ciudades y de los territorios más complejos y por ende integrarse a la geografía física y al análisis espacial y formal de los arquitectos, lograron la sumisión de estos enfoques. Así la forma quedó restringida a la escala de la arquitectura edilicia -más o menos voluminosa-, y la estética al objeto artístico, al objeto edilicio o al mobiliario urbano.

Los espacios públicos son concebidos por las administraciones de las ciudades como elementos aislados: se inauguran plazas y parques, no sistemas. Cuando los equipos técnicos municipales intentan incorporar una mirada de sistema a los parques, plazas, belvederes, caminos y calles por donde pasear, bordes de 


\section{ID I NVESTIG AC I O NES}

cursos de agua o frentes marítimos, se encuentran con el desafío de "coser retazos", muchos de ellos terrains vagues -o con menos poesía: terrenos abandonados-, dejados al dominio público como saldo del implacable crecimiento y aprovechamiento para loteos privados. Y el problema es más de calidad de los espacios, de posibilidad de su continuidad, de su morfología, de su articulación topológica y de sus proporciones, que de la cantidad de hectáreas disponibles.

Los historiadores de la arquitectura y del urbanismo han criticado -y no sin razón- al formalismo ingenuo, a los caprichos formales y a los dogmatismos estilísticos. $^{2}$ Entre otros factores, los excesos de formalismo han llevado, al fracaso de planes reguladores, directores e incluso de planes estratégicos, así como al urbanismo urbano y al pragmatismo de las intervenciones puntuales como único recurso "planificador".

Pero en ocasiones la crítica se transforma en ensañamiento contra la propia dimensión formal, la estigmatiza y, con ello, logra empobrecer la disciplina. Tal es el caso de Brasilia.

Si el núcleo fundacional de Brasilia constituye el paradigma por antonomasia de la forma hecha ciudad, las acusaciones a su inadecuación al crecimiento, a la dualidad social y a la falta de planificación estructural y formal posterior fueron tan demoledoras, que se convirtieron en una especie de demonización. Ambas, fundación y crítica marcaron la doctrina urbanística internacional.

\footnotetext{
${ }^{2}$ Básicamente me refiero a los autores de mayor adscripción a la modernidad en su crítica al formalismo academicista tales como Benévolo, Zevi, Gideon. No obstante ellos también cayeron en análisis de corte formalista respecto del urbanismo moderno. Un segundo aluvión de críticas al formalismo en el urbanismo se refiere a los límites y eventuales fracasos del urbanismo urbano como principal herramienta de planificación.
} 


\section{ID I NVESTIG AC I O NES}

Desde mi punto de vista, las críticas son en buena parte dogmáticas y superficiales. Quienes conozcan Brasilia podrán emocionarse percibiendo el arte en sus múltiples dimensiones, de corta y larga distancia, vivirán un espacio de máxima imaginabilidad al decir de Kevin Lynch, sentirán la fuerza del espacio existencial, al decir de Christian Norberg-Schulz, podrán recorrerla en secuencias propias al análisis pictórico de Phillipe Panerai y vivirán un paisaje cultural en que el(los) genio(s) del lugar podrán conmoverlos. Quienes "sobrevuelen" en el Google Earth los alrededores de Brasilia podrán constatar tejidos urbanos extensísimos perfectamente dibujados, estructurados, con tramas viales y morfologías de barrios diferenciados, diseñados, y verdaderos sistemas de espacios públicos que incorporan muy adecuadamente la hidrografía y las áreas naturales. En cualquier caso, nada tiene que ver la forma del trazado fundacional y de las arquitecturas de Brasilia con eventuales "fracasos" urbanísticos. Nadie ha podido fundamentar que la aglomeración de la Gran Brasilia sea peor que las demás metrópolis del mundo.

Se ha demostrado que la forma es indispensable para dar calidad a la ciudad, al territorio y a sus proyectos: teóricos, intervenciones urbanas y arquitectos preocupados por la forma y la excelencia.

Si los aportes de los teóricos de los años 60 y 70 tuvieron muchos adeptos, las repercusiones concretas, cuando exitosas y de calidad, fueron escasas, sin una trascendencia de magnitud suficiente, como para marcar sectores importantes de las ciudades. Las excepciones han sido mundialmente publicitadas como el caso de Barcelona. Operaciones de cirugía urbana mayor ocurren en ciudades que han tenido el coraje y la brillantez innovadora de técnicos y políticos que vieron en el proyecto urbano un medio para dar un salto cualitativo relevante, una bifurcación esperanzadora en un devenir decadente.

En las últimas dos décadas, las exploraciones proyectuales sobre los paisajes culturales, los territorios de la innovación, los territorios inteligentes, los compo- 
ID_INVESTIGACIONES

nentes urbanos de excelencia transitan por enfoques, métodos y modalidades de proyecto que revalorizan la dimensión espacial y formal de la herencia geográfica, urbana, patrimonial en múltiples escalas pero, fundamentalmente, recuperan las escalas meso y macro.

\section{NUEVE CASOS URUGUAYOS}

En Uruguay, en la última década y media, se han producido avances en este sentido insertos en procesos de elaboración de planes de ciudades y de regiones. $^{3}$

En los casos que se reseñan, la mirada del arquitecto busca poner en valor componentes de la forma subyacente. Esta tarea le es propia e intransferible a nuestra disciplina. En cada caso, aún en los más modestos, los mensajes del soporte geográfico y de la herencia patrimonial fueron parte de las principales orientaciones del plan.

A partir de ellos, se ensaya una categorización taxonómica que permite orientar el trabajo en futuros planes.

\subsection{Trinidad: la ciudad cuadrada}

Heredera de la modalidad de los trazados de las Leyes de Indias, vigentes en Uruguay hasta 1877, en que se establece el Decreto de Prescripciones para el trazado de nuevos pueblos y colonias en el territorio de la República, la Santísima Trinidad de los Porongos, Trinidad, se funda como un cuadrado de

\footnotetext{
${ }^{3}$ El autor se ha desempeñado como director y/o redactor de los planes analizados (salvo el caso de
} Montevideo), enmarcado en convenios Institucionales. 


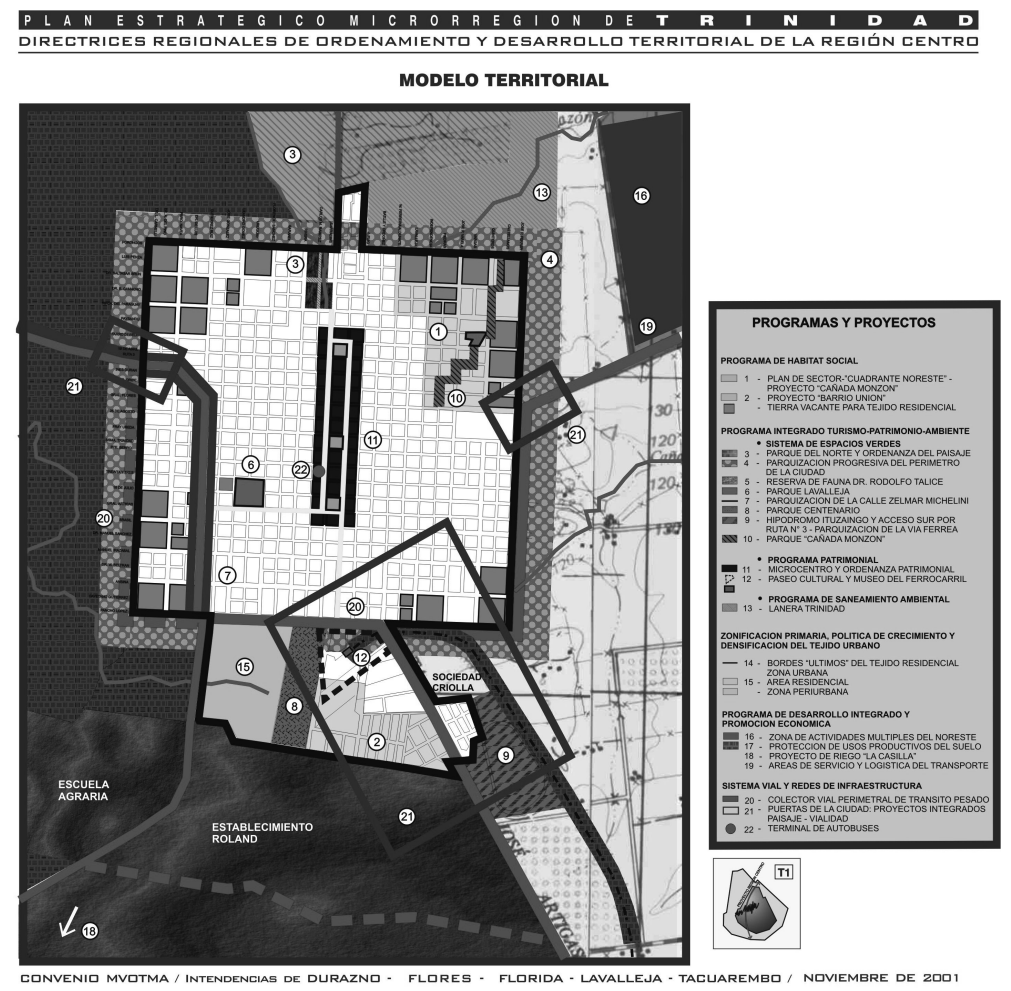

Trinidad, Plan estratégico. Fuente: DINOT/IM Flores (2001) p. 14

cuadrículas (2.400 metros de lado, 576 hectáreas brutas): un amanzanado interior en cuadrados de 100 metros de lado y una doble corona de manzanas cuatro veces mayores. El escaso crecimiento demográfico (21.000 habitantes $\left.{ }^{4}\right)$ de esta capital de un Departamento de ganadería ovina extensiva hace que, a

${ }^{4}$ Los datos de población corresponden a un redondeo del censo uruguayo de 1996. 


\section{ID_INVESTIGACIONES}

200 años de su fundación, el 15\% de su superficie loteada original, unas 85 hectáreas, aún no esté ocupada. Sólo una expansión al Sur y una estrecha expansión al Norte desbordan el cuadrado original.

En el Plan estratégico para la ciudad y su entorno, cuya primera versión es de 2001 y en el plan director de 2003, propuse poner en valor la geometría del plano fundacional, estableciendo un anillo verde de parquización progresiva libre de urbanizaciones- de 200 metros de ancho en todo el contorno rural aún no sobrepasado (DINOT/IM Flores 2001 y 2003). ${ }^{5}$ Complementariamente se consolida el amanzanado y cataloga el patrimonio construido del micro centro.

Luego de casi 10 años de los primeros bocetos, el Plan de enero de 2010 sostiene esta idea fuerza vinculada a la impronta formal original, apostando a consolidar la imagen de la única ciudad cuadrada del país y de la región. ${ }^{6}$

La geometría detectada como un contraste figura-fondo entre la matriz urbana fundacional y el campo agrícola se descubre como un componente patrimonial - la traza, el casco -, se evidencia como un componente del espacio perceptivo

\footnotetext{
${ }^{5}$ DINOT/IM Flores (2001) Plan Estratégico de Trinidad. Montevideo: Convenio MVOTMA/Intendencias de Durazno, Flores, Florida, Lavalleja, Tacuarembó (pag. 6). El Plan Director en sus aspectos normativos establecerá la Parquización Progresiva del perímetro de la ciudad, definiendo un cuadrado perfecto... como una imagen de marca de la ciudad. En el Plan Director de 2003 se establece: Artículo $16^{\circ}$. Zona de Desarrollo Proyectado por el Gobierno Departamental - Perímetro Parquizado ...se medirán doscientos metros hacia el exterior del área urbanizada de la ciudad a partir del límite exterior de la zona ZR3, Zona Jardín... uso agrícola, la superficie mínima para fraccionar en lotes será de 1 (una) hectárea y se establece un FOS máximo de $3 \% . .$. El proyecto paisajístico para esta franja perimetral será elaborado por la Intendencia en coordinación con los Organismos Públicos o Privados Nacionales o Internacionales vinculados a la agroforestía y parquización periurbana.

${ }^{6}$ Un Plan parcial está previsto para su instrumentación. Cabe señalar que en 2000 el Intendente Departamental al comprender este aspecto de la forma de su ciudad fue un vocero privilegiado de difusión de su potencial distintivo.
}

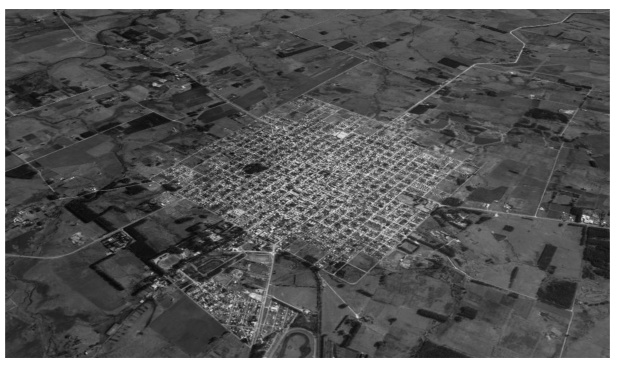

Ciudad de Trinidad, Fuente: Google Earth 
ID_I N VESTIG AC I O NES

desde puntos de vista aérea o de horizonte normal, y como una cualidad que, una vez materializada por el anillo parquizado, queda impregnada en el espacio concreto y en el espacio cognoscitivo.

\subsection{Paso de los Toros: capital de los lagos del centro}

Situada en el centro geográfico de Uruguay, Paso de los Toros (13.000 habitantes) se implantó sobre un estrechamiento del río Negro, antaño, pasaje de las tropillas de ganado vacuno, y sobre la ruta nacional $N^{\circ} 5$, espina dorsal de la vialidad nacional, que la conecta al Sur con Montevideo y al Norte con Rivera sobre la frontera con Brasil. Por ello, la Carta de desarrollo de la Región Centro (MVOTMA/Intendencias 2003) ${ }^{7}$ le adjudica el rol de "capital" de los lagos del centro.

A escasos $7 \mathrm{~km}$ de la represa hidroeléctrica de Rincón del Bonete, esta ciudad está marcada por su borde fluvial sur, sometida a los vaivenes de sus desbordes y retracciones. Es sobre estas costas que los ciudadanos y turistas de la zona disfrutan del esparcimiento y sufren de las inundaciones.

El sistema de espacios verdes constituyó la principal apuesta a la transformación del paisaje de la ciudad, a través de cinco piezas fundamentales: el Parque de la Costa del Río Negro, el Corredor Verde de Ruta $N^{\circ} 5$ (paisaje de ingreso calificado), el Parque de la Vía Férrea (sobre ramales abandonados vertebra el centro y consolida noreste), el Paseo de la Cañada Ceibal y el Eje del Norte.

${ }^{7}$ La Carta regional de desarrollo Región Centro (2003) fue elaborada en convenio MVOTMA/Intendencias de Durazno, Flores, Florida, Lavalleja, Tacuarembó. Documento inédito. 
ID_INVESTIGACIONES

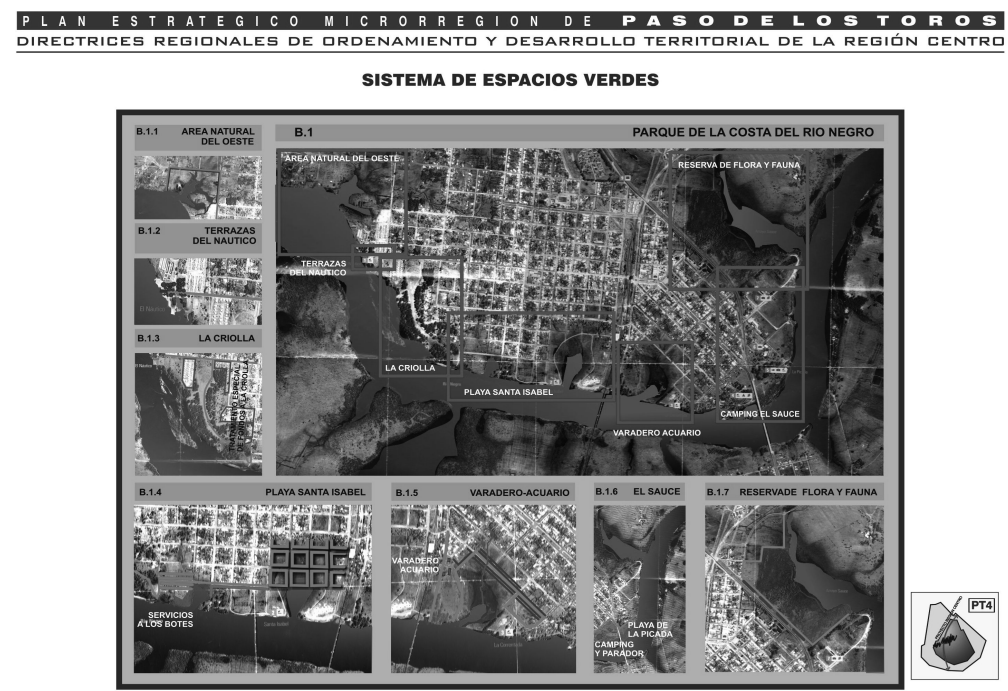

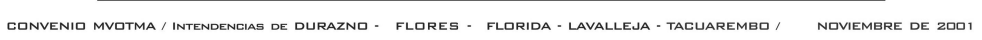

Paso de los Toros, Fuente: DINOT/IM Tacuarembó (2001) p. 20

El Plan Estratégico, aprobado en 2004, enfatiza el valor estructural de la faja costera, trabajándola en su lógica de conjunto de arco fluvial y en cada uno de sus tramos caracterizados: El parque de la Costa del río Negro conforma la envolvente principal del tejido urbano, y actúa como regulador de conflictos con la inundación. Como tal tendrá fases de ejecución progresiva con una gestión integrada. Está compuesto por una secuencia de espacios de esparcimiento ... (DINOT/IM Tacuarembó 2001). ${ }^{8}$

${ }^{8}$ DINOT/IM Tacuarembó (2001) Plan Estratégico de Paso de los Toros. Montevideo: Convenio MVOTMA/Intendencias de Durazno, Flores, Florida, Lavalleja, Tacuarembó. (pag. 7 y 8) ...siendo los proyectos específicos, de Oeste a Este: Area Natural del Oeste, Terrazas del Náutico, La Criolla, Playa Santa Isabel, Varadero. Acuario, Camping El Sauce y Parque de la Playa, La Picada, Reserva de Flora y Fauna. 
La hidrografía y el patrimonio ferroviario son los inspiradores del sistema de espacios públicos, y constituyen un componente sustantivo de la propuesta urbanística, reafirmando el espacio vivencial costero y enriqueciéndolo al conectarlo tejido "adentro" en el paseo de las vías.

\subsection{San Gregorio de Polanco: ciudad de arte sobre el espejo del lago}

Pequeño poblado rural (3.000 habitantes), vio acercarse a sus casas el agua del río Negro cuando la represa Rincón del Bonete se iba llenando. La silueta de su contorno cambió drásticamente, transformándose en una insospechada y original península urbana con un apéndice forestado de $3 \mathrm{~km}$ por 600 metros.

Su plano fundacional, cuadriculado, fue dotado por el Agrimensor de Número Cornning en $1852^{\circ}$, con un componente distintivo como principal espacio público: una faja en forma de gradiente. Este cinturón verde "amuralla" la ciudad creando un recinto de gran originalidad. Pese a que ha sido mutilado $y$ fragmentado con construcciones públicas, constituye aún un patrimonio urbano único susceptible de ser puesto en valor fácilmente en su trazado general, aunque seguramente no con la contundencia de su gesto creador. Para ello el Plan Estratégico establece una serie de acciones de plantación, ajardinado y equipamientos en este cinturón verde concebidas en un proyecto unitario y de ejecución progresiva. (DINOT/IM Tacuarembó 2001). ${ }^{10}$

El diálogo entre esta forma escalonada y el borde sinuoso y cambiante de la costa es puesto en valor en el Plan Estratégico aprobado en 2004. Un muro de contención rectilíneo subraya un kilómetro del borde Sur del lago, completando

\footnotetext{
${ }^{9}$ Topógrafo

${ }^{10}$ DINOT/IM Tacuarembó (2001) Plan Estratégico de San Gregorio de Polanco. Montevideo: Convenio MVOTMA/Intendencias de Durazno, Flores, Florida, Lavalleja, Tacuarembó. (pag. 7)
}
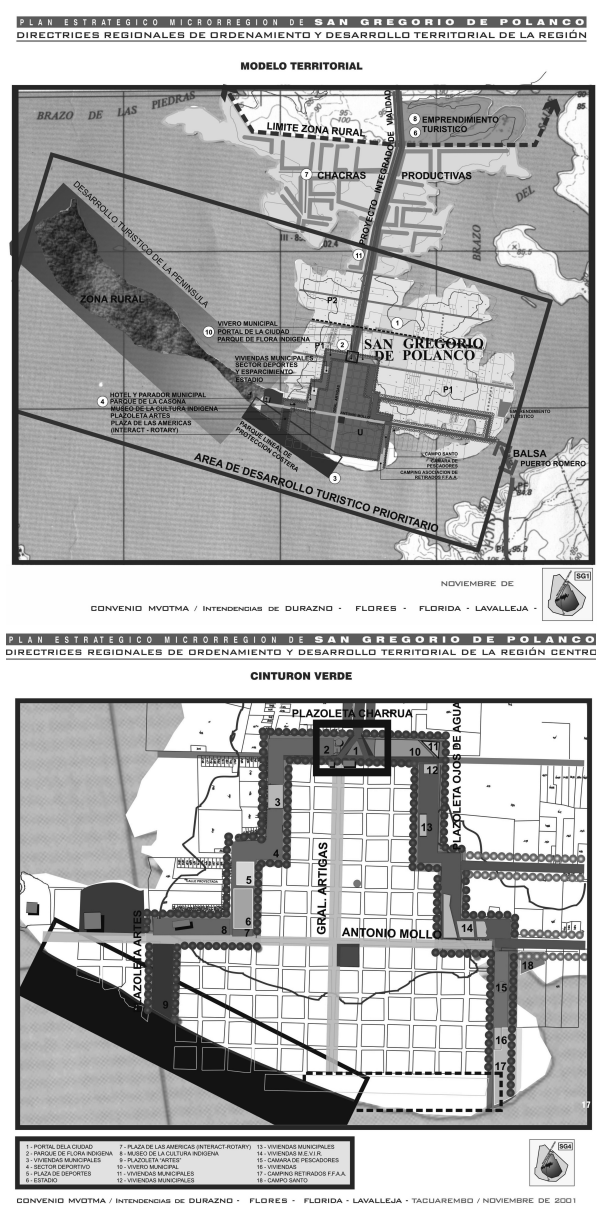

San Gregorio de Polanco, Fuente: DINOT/IM Tacuarembó (2001), 14 y 17 


\section{ID I NVESTIG AC I O NES}

la singularidad del contorno de la ciudad. Pinturas murales del reconocido taller del artista uruguayo Clever Lara, aplicados en casas, comercios y muros medianeros, salpican las calles de arte (primer museo abierto de artes visuales del país). A pocos metros del centro o, a veces, en pleno centro, alguna oveja, vaca o gallina completa un curioso y muy atractivo genio del lugar. El cementerio queda aislado por el agua del embalse en numerosas ocasiones. EI lago, las playas, un gran camping, la inmensa península forestada, pescadores artesanales y una balsa que posibilita el cruce al vecino Departamento de Durazno completan la escena. Un Programa Gerencial del Paisaje de la Cultura Local da cuenta de ello: Conjunción única en el país de paisaje lacustre y rural, arte público y escala urbana pequeña, el atardecer en el lago, playas y barrancos, artesanía local y gastronomía de campaña, patrimonio construido como expresión local: Ciudad de Arte sobre el Espejo del Lago. (DINOT/IM Tacuarembó 2001). ${ }^{11}$

Tres elementos de geometrías contrastantes dialogan conformando una impronta de alta singularidad: una franja de espacio público en forma de rédent que enmarca el conjunto de la cuadrícula urbana, la ondulada y oscilante línea de costa del lago y el murallón rectilíneo de defensa costero de grandes dimensiones. Geografía y herencia patrimonial marcan al poblado; los habitantes con sus manifestaciones culturales enriquecen, realzan, modelan y

\footnotetext{
${ }^{11}$ DINOT/IM Tacuarembó (2001) Plan Estratégico de San Gregorio de Polanco. Montevideo: Convenio MVOTMA/Intendencias de Durazno, Flores, Florida, Lavalleja, Tacuarembó. (pag. 5). El sistema de espacios verdes constituye la principal apuesta a la consolidación, protección y mejora del paisaje. El mismo se compone de seis piezas fundamentales: la Ordenanza General del paisaje, la Ordenanza de Protección Costera, la Fachada Costera, el Cinturón Verde, el Parque Lineal de Protección Costera, el Parque de la Casona. DINOT/IM Tacuarembó (2001) Plan Estratégico de San Gregorio de Polanco. (pag. $5)$.
} 
ID_INVESTIGACIONES

construyen su paisaje cultural con multiplicidad de formas, colores y actividades.

3.4 Fray Bentos: un enclave de diseño inglés en un territorio de lógica española

La apuesta del Plan de Fray Bentos para un desarrollo innovador y endógeno (frente a la gran usina de celulosa de la empresa Botnia) se centra en el Sistema Patrimonial Industrial Anglo-Liebig/SPIA.

Sobre la costa del río Uruguay, el imponente edificio de sus cámaras frigoríficas ( $100 \times 50 \times 50$ metros) y su chimenea dominan el skyline de la ciudad y de la costa aguas abajo. Multiplicidad de construcciones albergan todo el ciclo productivo de la carne desde la matanza al procesamiento del ganado altamente industrializado. Primero en la fase de saladero Liebig, luego con los mundialmente famosos extractos y conservas Fray Bentos y Oxo, se desarrolló un sistema complejo y completo. Sánchez Rossel (2007) sistematizó los componentes del SPIA incluyendo la costa, las estructuras portuarias, la conectividad ciudad-complejo Anglo-unidades de producción, barrio inglés, instalaciones deportivas, corrales, construcciones e Instalaciones industriales de las fases de saladero y de frigorífico (Liebig, Anglo), trazas, sitios de festividades, recorridos de animales y personas, y elementos del territorio que pertenecen al sistema pero que están fuera del núcleo. ${ }^{12}$

La impronta inglesa caracteriza el paisaje y abarca la implantación, los trazados urbanos, las edificaciones y sus detalles constructivos.

12 SANCHEZ ROSSEL, Astrid (2007) Orientaciones estratégicas para el plan director del Sistema Patrimonial Industrial Anglo. Montevideo: IMRN (pag. 22) Se identifican los siguientes componentes del Sistema Patrimonial Industrial ANGLO 


\section{ID I NVESTIG AC I O NES}

Se ha puesto en marcha un Plan de valorización y gestión del SPIA con la ambición de ingresar en el selecto grupo del patrimonio de la Humanidad de UNESCO. La dimensión paisajística de la propuesta es clave y hace fundamental hincapié en las cuencas visuales, en los trazados, en la morfología de las futuras actuaciones en su entorno y en el respeto por las formas.

Si la singularidad del río Uruguay es definitoria del paisaje, de la ciudad y de su implantación, el Patrimonio industrial percibido y valorizado como sistema es la apuesta sustantiva del Plan, una herencia patrimonial que ha de tomar una multiplicidad de dimensiones: desarrollo local, espacio existencial reafirmado, paisaje cultural con una escala ampliada a la cuenca baja del río y a su transnacionalidad.

\subsection{Minas: la ciudad de la serranía}

Enclavada en un pequeño valle en las serranías, Minas tiene su atractivo fuera, en sus alrededores inmediatos, en el marco en que se implanta.

Esta ciudad, que intenta vivir desde hace décadas de las migajas del turismo de Punta del Este mediante una alternativa a la "oferta" de sol y playa de la costa Atlántica, ve peligrar el atractivo de sus sierras pedregosas y escarpadas por un manto de eucaliptos que oculta su esencia e identidad, homogeneizando el conjunto.

La forestación ha avanzado rápidamente sobre las serranías al influjo de una ley de promoción apoyada, desde fines de los años 1980, sobre estos tipos de suelos en que la agricultura es inviable y el pastoreo dificultoso. El Estado central no ha sabido razonar con dimensión espacial ni con estrategias de desarrollo coherentes en los territorios serranos en los que convergen al menos tres ramas de la economía: el turismo, la forestación y la minería. 


\section{ID_INVESTIGACIONES}

En el Plan Estratégico establecimos al paisaje como principal fortaleza; procura la protección de las serranías en el entorno inmediato y en un área de influencia mayor. Para ello se propone la creación de un Parque Regional Turístico y Patrimonial de las Sierras apoyado en la valorización de su patrimonio ambiental, paisajístico, cultural, arquitectónico, arqueológico y productivo, integrando a los actores públicos y privados involucrados en la instrumentación de productos turísticos regionales... El Parque tiene cuatro grandes componentes: un territorio, una organización aplicada sobre éste, un programa de acción y una normativa territorial y ambiental... El Plan Estratégico establece como Territorio Provisional del Parque los ámbitos siguientes: la Microrregión de Minas (Aguas Blancas y las Sierras de Minas, Arequita, Villa Serrana y Penitente), Asperezas o Callosidades de Polanco y la formación Geológica de la Sierra de la Ballena (DINOT/IM Lavalleja 2001). ${ }^{13}$ Asimismo se prevé una Ordenanza General del Paisaje.

La forma a valorizar es, para el caso, directa y simplemente el relieve, el soporte natural, en su estado más natural posible. La geografía física es la protagonista.

\subsection{Rivera: el espacio del Entre-deux}

Rivera, ciudad de frontera seca con Brasil, fue fundada para instalar y perennizar el Entre dos -el Entre-deux al decir de Bernard Ecrement (2010). ${ }^{14}$ Junto a Santana do Livramento, su tejido urbano se extiende sobre una sierra

\footnotetext{
${ }^{13}$ DINOT/IM Lavalleja (2001) Plan Estratégico de Minas. Montevideo: Convenio MVOTMA/Intendencias de Durazno, Flores, Florida, Lavalleja, Tacuarembó. (pag.7 y 8 ).

${ }^{14}$ ECREMENT, Bernard (2010) Les territoires de l'entre-deux, en : Revista F@ro Número 12, Playa Ancha Chile
} 


\section{ID I NVESTIG AC I O NES}

escarpada bajo la forma de una cuadrícula "mandatada" por la autoridad fundadora desde la capital del país, quinientos kilómetros al Sur.

El plan de Rivera juega sus principales apuestas de espacios públicos en los espacios compartidos: "la línea" (calle y plaza donde dialogan ambas ciudades), el sistema de vías férreas que se interna sinuoso en el tejido de Santana, los cerros de frontera (del Norte, del Marco y Caqueiro) y el eje comercial, que se mantiene a uno y otro lado.

Aún antes de la aprobación de su Plan en 2010, el Gobierno de la ciudad está valorizando sus espacios centrales de mayor carga simbólica y valor de uso para la cultura local y pequeños espacios, estratégicamente seleccionados. Un diseño innovador, de excelencia, pone el toque de singularidad a cada sitio, con materiales de la región (piedra arenisca, madera dura y piques de granito) como si el genio del lugar le susurrara al oído para desencadenar su genio creativo. $^{15}$

El diseño de los espacios del Entre-deux, que afianza la histórica integración transfronteriza, se aplica también a espacios de integración entre la Rivera tradicional y los barrios fragmentados por los cursos de agua en la Rivera nueva, al otro lado del arroyo Cuñapirú y sus áreas de inundación.

Un tercer espacio del Entre-deux toma una dimensión subcontinental: el acuífero Guaraní. Rivera, situada en un punto neurálgico de la fina faja de recarga de este gigantesco acuífero que abarca el norte uruguayo, parte del Norte argentino, el Este de Paraguay e importantes territorios de Brasil, asume

\footnotetext{
${ }^{15}$ Diseños y dirección de obra del arquitecto Leonidas Bayo.
} 
su responsabilidad en el plan urbano proponiéndose como laboratorio para su gestión y manejo ambiental.

El Programa turismo-patrimonio-ambiente (LIGRONE y otros 2004) ${ }^{16}$ del Plan de Rivera aprobado en 2010 por las autoridades nacionales, establece ocho componentes principales: Programa Ambiental Acuífero Guaraní, Programa Ambiental Cuenca del Cuñapirú, Sistema de Espacios Verdes, bordes parquizados entre el tejido y el Cuñapirú, Avenidas y Calles Parquizadas, Programa y Proyecto "la línea", Rivera arte y cultura y el Proyecto Arte sobre rieles.

En suma, el plan de Rivera juega sus apuestas sobre mensajes de la geografía en escala subcontinental (su rol en el acuífero Guaraní), en la escala local con su hidrografía y relieve de cerros y sobre los espacios culturales originados en el entre-deux fronterizo y el sistema de espacios públicos que tiende a enlazar los tejidos de ambas ciudades y sus barrios.

\subsection{Los parques patrimoniales del Área Suroeste}

El proyecto de desarrollo y ordenamiento territorial del Área Suroeste abarcó los Departamentos de Colonia, San José y Soriano. Entre sus estrategias, un capítulo relevante refiere a la puesta en valor de la tríada Turismo - Patrimonio - Ambiente (LIGRONE y otros 2009). ${ }^{17}$

Para comprender el espacio turístico regional y diseñar las estrategias para su desarrollo resultó fundamental establecer la estructura y morfología de los

\footnotetext{
${ }^{16}$ LIGRONE, Pablo y otros (2004). Plan de desarrollo y ordenamiento territorial de la microrregión de Rivera. Disponible en: www.rivera.gub.uy (Art. 15 pag. 13)

${ }^{17}$ LIGRONE, Pablo y otros (2009) Directrices Regionales de Ordenamiento y Desarrollo Territorial del Area Suroeste - Programa Integrado Turismo-Patrimonio-Ambiente. Montevideo: DINOT/MVOTMA Cuadernos del territorio $\mathrm{N}^{\circ} 3$
}

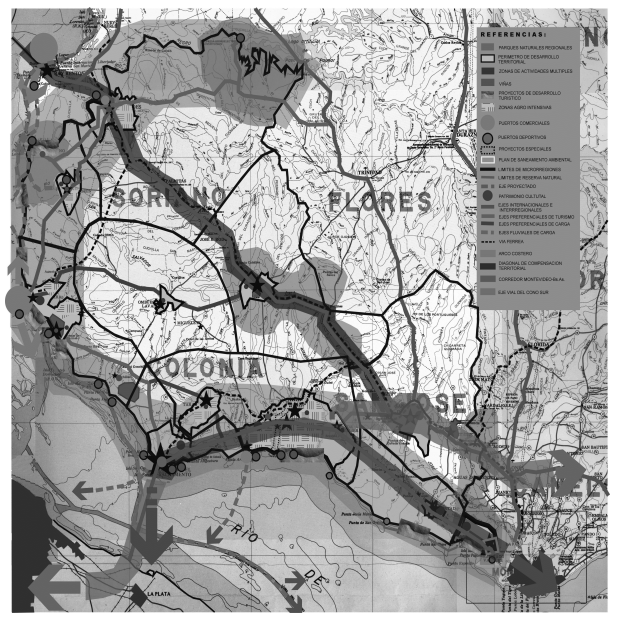

Directrices estratégicas para el Área Suroeste. Fuente: Pablo Ligrone y otros (2006) p. 45 


\section{ID I NVESTIG AC I O NES}

atractivos turísticos actuales y potenciales, las unidades, complejos, centros, corredores, zonas y subzonas turísticas.

Cinco importantes piezas territoriales fueron identificadas, delimitadas y descritas. Cada una de ellas fue asociada a un instrumento de gestión apropiado a su valorización: los parques patrimoniales:

- Las sierras de Mahoma y Mal Abrigo.

- El río Santa Lucía inferior.

- La cuenca media e inferior del Río Rosario.

- Las desembocaduras de los Ríos Negro y San Salvador.

- El lago Palmar.

Estas piezas y la lectura de conjunto del arco costero del Río de la Plata y del arco fluvial del Río Uruguay y Río Negro, asociada a las rutas litorales e interiores, han puesto en evidencia la estructura, en ciernes, de una estrategia de valorización de espacios naturales y culturales que pueden convertirse en productos turísticos.

La geografía leída bajo el prisma de los paisajes culturales y de los productos turísticos de base paisajística y patrimonial es el método a través del cual hemos descubierto un territorio vasto y diversificado. Las formas así dibujadas permiten mostrar los potenciales de desarrollo a escalas macro, meso y micro, pudiendo enlazarlas entre sí, flexiblemente, en función de objetivos de desarrollo cambiantes.

\subsection{Región de los Lagos del Centro}

Los embalses de las represas hidroeléctricas de Rincón del Bonete, Baygorria y Palmar conforman la región de los Lagos del Centro. Una secuencia de saltos del Río Negro se domestica para optimizar el recurso energético. Desde la represa Palmar, aguas abajo, hasta el extremo Este del Bonete, 170 kilómetros de espejos de agua de anchos hasta ramales de $20 \mathrm{~km}$ a un lado o al otro del 


\section{ID I NVESTIG AC I O NES}

curso principal, marcan al territorio uruguayo: el centro fragmenta al Norte y al Sur del país. Esta singularidad es la única de dimensiones relevantes de un extenso mundo rural nacional.

El paisaje resultante tiene múltiples atractivos escénicos: vastos espejos de agua, orillas de monte criollo, plantaciones de pinos, playas de arenas limpias e infinitas perspectivas generadas por meandros y ramales. Solamente son accesibles las costas correspondientes a los poblados de Palmar, Baygorria y Bonete, vinculadas a la construcción de las represas y a su gestión posterior, a los pequeños parques Andresito y Bartolomé Hidalgo junto al puente de la ruta $\mathrm{N}^{\circ} 3$, Paso de los Toros y pueblo Centenario, junto al puente de la ruta $\mathrm{N}^{\circ} 5 \mathrm{y}$ San Gregorio de Polanco, en el lejano y extremo Este.

La escasa actividad turística se concentra en estos puntos y consiste, mayoritariamente, en esparcimiento natural y descanso para la población de las zonas cercanas.

El Proyecto de desarrollo y ordenamiento territorial para la Región Centro elaboró un dossier de los Lagos que tiene por ambición divulgar sus atractivos y sus potenciales. Se mostró a las autoridades nacionales y departamentales que esta región de lagos constituye una unidad con forma propia, con una lógica sistémica que va más allá de la explotación energética del recurso agua. Esta pieza de la geografía nacional es un diamante en bruto, un territorio cuya forma y tamaño es excepcional y amerita múltiples desarrollos.

\subsection{Montevideo: ¿la bahía?}

Montevideo es una hermosa ciudad, pero no por mérito reciente sino por herencia de un pasado de opulencia económica y de esplendor cultural; luego de los años 1950, los Gobiernos Departamentales han intentado administrar la decadencia derivada de la combinación del despilfarro del espacio (expansión en bajísima densidad), de la pobreza urbana y de la falta de innovación 


\section{ID_INVESTIGACIONES}

planificada. Los espacios públicos, de diseño burocrático y excepcionalmente concursados, no han llegado, a las dimensiones y calidades de los tres grandes espacios de referencia histórica: la Rambla Sur (borde costero), el parque Rodó y el parque Batlle y Ordóñez (parques de importantes dimensiones con respecto al tamaño de la ciudad). En los tres se evidencia la alta calidad del diseño, de las formas, de la hechura y de genio artístico.

En las últimas décadas, la preocupación por el espacio público puede "medirse" en unos cientos de metros de peatonalización en la Ciudad Vieja, en algunas plazas y rincones dispersos, en un tramo de recuperación del arroyo Miguelete y en unos cortos segmentos de Rambla costanera en el barrio del Cerro. Estas acciones surgieron de un accionar filosóficamente pragmático.

Si la obra pública montevideana de los últimos quince años no puede ser presentada como un éxito del manejo del espacio público en la actuación de detalle, como quisieron sostener los defensores del "pragmatismo caso a caso" como alternativa al "idealismo" planificador, el Plan Montevideo de 1998, en revisión actualmente, tampoco puso el énfasis en ello. Por lo tanto, no encontramos actuaciones en piezas relevantes del espacio público montevideano que provoquen impactos formales-espaciales-estéticos significativos.

Sin embargo, es de destacar la importancia que el Plan Montevideo adjudica al entorno de la bahía, calificada por éste como área de promoción, y la expectativa que generó al anunciar la planificación derivada en barrios de valor patrimonial (Ciudad Vieja, Pocitos, Barrio Sur, Carrasco-Punta Gorda, PradoCapurro y Colón Lezica) y planes especiales (Fénix-La Aguada-Bella Vista y el Arroyo Miguelete junto a la bahía).

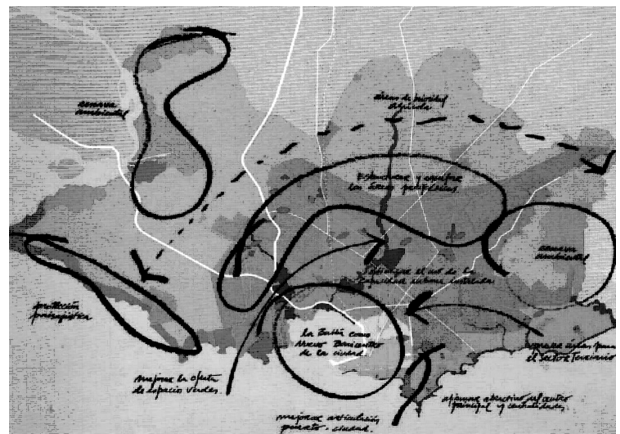

Montevideo, Avance del Plan de Ordenamiento Territorial (1998-2005). Fuente: IMM (1997) p. 21

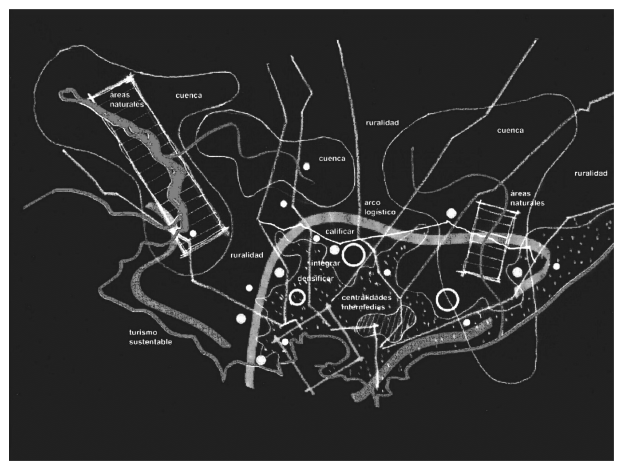

Montevideo, Plano Síntesis de Ordenación 20102020, Fuente: Avance Revisión del Plan Montevideo, IMM (2010) p. 85 


\section{ID_INVESTIG AC I O NES}

La bahía de Montevideo, junto con el Cerro y la Rambla Sur, son los tres principales componentes geográficos, símbolos de referencia para los ciudadanos y para todo el país.

El asunto "borde costero" se convirtió, desde la Barcelona Olímpica, en un motivo de reconversión urbana a la moda. Toda ciudad que pudo, actuó sobre un tramo de water front. Montevideo, que ya contaba con más de $20 \mathrm{~km}$ de borde costero con murallones de granito rosado, playas, amplios paseos, aceras y espacios verdes y un bulevar que canaliza uno de los principales flujos del país, todo ello disfrutable por toda la población uruguaya libre y masivamente, se contagia también de esta moda. La Intendencia, el Banco Hipotecario del Uruguay, no pocos arquitectos municipales y profesores universitarios quieren promover para Montevideo un "Puerto Madero", implementado en la renovación costera de la vecina ciudad de Buenos Aires. ¿Dónde? En el peor lugar: en el borde urbano frentista al puerto comercial de aguas profundas, el único que parece tener Uruguay. La iniciativa, bautizada como Plan Fénix, quedó en cenizas aún antes de nacer. En otro tramo, años más tarde, el Plan de la Ciudad Vieja parece insistir con que el puerto comercial se "abra" al deambular de paseantes.

Quienes promueven la idea de "recuperar todo el frente costero de la bahía para la ciudad" parecen suponer que el puerto comercial, principal equipamiento del país y sin el cual Uruguay no hubiera existido ni existiría, es ajeno a la ciudad, una traba a superar para que los habitantes puedan llegar al borde de las contaminadas aguas de la bahía, eludiendo grúas, camiones y contenedores y así poder disfrutar de los bordes de los muelles... como si los $20 \mathrm{~km}$ de paseo ya mencionados no alcanzaran. Felizmente, la cordura más recientemente focaliza en un sector viable del frente costero en el Plan Capurro Bella Vista. 


\section{ID I NVESTIG AC I O NES}

Estas ideas de los años 1990 fueron una buena excusa para que, justamente en 1998, el primer Seminario Montevideo, seminario anual de carácter internacional, tuviera como tema central de proyecto urbano "la Bahía".

El producto de este seminario, que puso a reflexionar y proyectar a un importante grupo de profesores y estudiantes de la Facultad de Arquitectura, fue muy importante por la calidad y diversidad de las conceptualizaciones, enfoques, métodos y propuestas.

Todos los talleres, dirigidos por destacadísimos arquitectos ${ }^{18}$, tomaron a la bahía en su conjunto y los tejidos urbanos adyacentes, poniendo en cuestión las problemáticas y desafíos de toda la ciudad, incluyendo su dimensión metropolitana.

Nos interesa destacar dos proyectos que concibieron la bahía como una unidad formal de gran escala, como un espacio unitario más allá de la diversidad de sus sectores circunvecinos.

Humberto Eliash propuso envolver el espejo de agua de la bahía en una elipse. La memoria del proyecto fundamenta extensamente la opción formal y unitaria:

Poética de la geografía y la geometría. El proyecto asume la bahía como un espacio geométrico de fuerte pregnancia formal que aprovecha la potencialidad de su geografía privilegiada. La elipse, forma perfecta de las leyes de Kepler, sublima el dramatismo de la búsqueda éticamente moderna de ordenar el caso y consolida, en la clara conciencia de la flecha del tiempo y de irreversible construcción de la ciudad, la ilusión del lugar eterno. Una ilusión capaz de asumir la referencia existencial colectiva dándole unidad a la bahía, definiendo

\footnotetext{
${ }^{18}$ Los directores de taller fueron Miguel Baudizzone de Argentina, Humberto Eliash de Chile, Iñaki Abalos y Juan Herreros de España, Hiroshi Hara de Japón, Paulo Mendes Da Rocha de Brasil, Carlos VillanuevaBrandt de Inglaterra
} 


\section{ID_INVESTIGACIONES}

un adentro "plaza" de aguas calmas, con alto contenido simbólico, y un afuera fragmentario con dinámicas a velocidades múltiples. La elipse une al hombre con el hombre a través de una cadena infinita de manos. La elipse -pista, paseo, borde, secuencia de faros-, al unir los fragmentos dialoga con ellos y genera oportunidades a su dinámica: genera tierra urbana, da forma al abandono, hace público el espejo de agua. Los diferentes episodios urbanos, escalas y paisajes desafían a la elipse y son por ella desafiados...En suma, la elipse revoluciona el ordenamiento de la bahía materializando el mito del país y construye la imagen de marca que nos posiciona en el siglo XXI. (ELIASH y otros 1998$)^{19}$

Paulo Mendes Da Rocha propuso envolver conceptualmente los tejidos urbanos linderos a la bahía con un cuadrado que permite visualizarla como una gran plaza, como un espacio unitario en que esos tejidos dialogan con la lámina de agua:

Generalidades... La bahía de Montevideo releva de inmediato, en el ámbito de la arquitectura, la idea de la dimensión técnica y la idea de disposición espacial, en cuanto forma e invención. La cuestión exige una firme contribución de ciencia, técnica y arte. La bahía ya es y será siempre una naturaleza humanizada, otra. La bahía, ahora, será una construcción. En las premisas el trabajo enfatiza en el examen de conjunto, en la espacialidad paisajística, geométrica, geomorfológica, entre la ciudad y la bahía, el tamaño de la bahía, la virtud de las aguas, la conectividad con las áreas interiores, la identidad cultural y la idea de "una ciudad para todos"... La propuesta tiene como objetivo acciones de transformaciones físicas que sean soporte de acciones

${ }^{19}$ ELIASH, Humberto y otros (1998) Taller Eliash en: Primer Seminario Montevideo - Bordes Urbanos. Montevideo: El Arqa (pag. 21 y 22) 


\section{ID I NVESTIG AC I ONES}

transformadoras de la vida de la ciudad, con amplia generación de espacios públicos de interlocución con la lámina de agua. Una visión, primaria, de totalidad de diseño de la bahía, en cuanto problema complejo y multidisciplinario. La bahía como un gran ingenio: ciudad naturaleza... (MENDES DA ROCHA y otros 1998) ${ }^{20}$

Mientras el plan de Montevideo de 1998 diseña su logo o imagen corporativa a través de un esquema del mapa de la ciudad en que el gesto de una elipse marca la bahía, el documento que en 2010 se puso de manifiesto público redibuja la bahía como prioridad estratégica, esta vez a la manera del cuadrado... ¿Coincidencias?

\section{CONCLUSIÓN}

Los ejemplos de planificación presentados muestran la relevancia de la tarea del arquitecto y su responsabilidad en la captación de las formas a escalas múltiples. No se trata de formas surgidas de un diseño ex-novo, con todo el arte y arbitrariedad que puedan éstas conllevar. Se trata de captar, en lo existente, aquello que estructural y morfológicamente pueda ser relevante para la transformación y la gestión de territorios concretos.

En todos los casos estudiados, las formas puestas en evidencia tienen la fuerza potencial de alimentar la imaginabilidad de cada ciudad, región o porciones de éstas. El solo hecho de darse cuenta que una ciudad tiene la originalidad de ser cuadrada o que existe una región de los Lagos del Centro o que la bahía de Montevideo puede ser leída como una gran plaza patrimonio

${ }^{20}$ MENDES DA ROCHA, Paulo. y otros (1998) Taller Mendes Da Rocha en: Primer Seminario Montevideo - Bordes Urbanos. Montevideo: El Arqa (pag. 28 y 29) 


\section{ID I NVESTIG AC I O NES}

de todos los uruguayos, supone un aporte sustantivo, que opera en la cultura de colectivos locales, nacionales o internacionales. Una utilidad inmediata de tales "descubrimientos" o "invenciones" de formas es la promoción turística de cada territorio al integrarse éstos a su imagen de marca.

La Geografía Física muestra ser una cantera muy fructífera de formas:

- Un arco costero como una unidad y sus segmentos caracterizados como en los casos de Paso de los Toros, el Área Suroeste o la bahía de Montevideo.

- Una unidad de relieve como paisaje caracterizado en sus valores escénicos y ambientales como en los casos de Minas y su entorno de sierras, las sierras de Mahoma y Mal Abrigo o los cerros de frontera en Rivera.

- Un cuerpo de agua como cuenca y como unidad perceptiva como en los casos del acuífero Guaraní y el arroyo Cuñapirú en Rivera, los lagos del Centro y el espejo de la bahía de Montevideo.

El Patrimonio urbano, arquitectónico y natural es otro de los orígenes privilegiados de la forma:

- Los trazados fundacionales que han pervivido y que son redescubiertos como en el caso de San Gregorio de Polanco o Trinidad.

- Edificios, sitios, obras de arte y monumentos abundantes en un área o de magnitud suficiente para imponerse en el paisaje como en los casos del

- Parque de la cuenca media e inferior del Río Rosario, San Gregorio de Polanco y Fray Bentos.

- Los componentes naturales y culturales integrados en paisajes caracterizados como los Parques del Área Suroeste y la bahía de Montevideo. 


\section{ID I NVESTIG AC I O NES}

El Entre-deux es la tercera de las fuentes de la forma a destacar:

- Los espacios de integración en áreas de frontera como en el caso de Rivera.

- Los lugares de construcción de cohesión social y cultural como en los casos de Rivera y de la bahía de Montevideo

Investigaciones basadas en las estructuras antropológicas y la fenomenología del espacio (la percepción, la vivencia, la internalización cultural, la carga simbólica de estas formas) pueden arrojar nuevas enseñanzas sobre el impacto de la metodología de proyecto y planificación estudiada.

En Uruguay, país sin tradición planificadora, quedan por identificar muchos componentes con potencialidad para convertirse en focos de desarrollo: el patrimonio ni se ve ni se valoriza y es considerado por muchos decisores como un problema; los ambientalistas son vistos como un freno para el desarrollo, por lo que los componentes del paisaje natural y en especial su valor escénico no tienen peso a la hora de optar entre su protección o el incremento del producto bruto; el turismo de sol y playa y las termas son los únicos argumentos turísticos de peso.

La tríada Turismo-Patrimonio-Ambiente, incorporada a los instrumentos de planificación, tiene la ambición de convertirse en un paradigma que construya una sinergia transformadora, revolucionaria en el urbanismo, el ordenamiento territorial y el desarrollo sostenible.

Deberíamos volver a llamar la atención sobre grandes piezas urbanas o territoriales, no con intenciones megalómanas sino con la idea de descubrir y poner en valor componentes olvidados y degradados, descubrir nuevas relaciones y jugar con y en la geografía física y los patrimonios culturales, reescribiendo, redibujando el espacio público a escala de toda la ciudad. 
ID_I N VESTIG AC I O NES

Nos interesa, en suma, poder captar los mensajes que lo heredado nos transmite, para escucharlos y entenderlos, con los sentidos abiertos a la creación, la innovación, la forma, la estructura, el sistema de espacios públicos y la estética.

\section{BIBLIOGRAFÍA}

DINOT/IM Flores (2001). Plan Estratégico de Trinidad. Montevideo: Convenio MVOTMA/Intendencias de Durazno, Flores, Florida, Lavalleja, Tacuarembó

DINOT/IM Flores (2003). Carta regional de desarrollo Región Centro. Montevideo: Convenio MVOTMA/Intendencias de Durazno, Flores, Florida, Lavalleja, Tacuarembó. Documento inédito.

DINOT/IM Tacuarembó (2001). Plan Estratégico de Paso de los Toros. Montevideo: Convenio MVOTMA/Intendencias de Durazno, Flores, Florida, Lavalleja, Tacuarembó

DINOT/IM Tacuarembó (2001). Plan Estratégico de San Gregorio de Polanco. Montevideo: Convenio MVOTMA/Intendencias de Durazno, Flores, Florida, Lavalleja, Tacuarembó.

DINOT/IM Lavalleja (2001). Plan Estratégico de Minas. Montevideo: Convenio MVOTMA/Intendencias de Durazno, Flores, Florida, Lavalleja, Tacuarembó. (pag.7 y 8).

ECREMENT, Bernard (2010). Les territoires de l'entre-deux, en : Revista F@ro Número 12, Playa Ancha Chile: Universidad de Playa Ancha, ISSN 0718-4018. Disponible en http://web.upla.cl/revistafaro/n12/pdf/art03.pdf

ELIASH, Humberto y otros (1998). Taller Eliash en: Primer Seminario Montevideo - Bordes Urbanos. Montevideo: El Arqa (pag. 21 y 22).

Intendencia Municipal de Montevideo, (1997) Avance del Plan de Ordenamiento Territorial (1998-2005), Montevideo: IMM

Intendencia Municipal de Montevideo, (2010) Revisión del Plan Montevideo, Montevideo: IMM 
ID_INVESTIGACIONES

LIGRONE, Pablo y otros (2004). Plan de desarrollo y ordenamiento territorial de la microrregión de Rivera. Disponible en: www.rivera.gub.uy.

LIGRONE, Pablo y otros (2006). Directrices Regionales de Ordenamiento y Desarrollo Territorial del Area Suroeste. Montevideo: DINOT/MVOTMA Cuadernos del territorio № 1.

LIGRONE, Pablo y otros (2009). Directrices Regionales de Ordenamiento y Desarrollo Territorial del Area Suroeste - Programa Integrado Turismo-Patrimonio-Ambiente. Montevideo: DINOT/MVOTMA Cuadernos del territorio $\mathrm{N}^{\circ} 3$.

MENDES DA ROCHA, Paulo. y otros (1998). Taller Mendes Da Rocha en: Primer Seminario Montevideo - Bordes Urbanos. Montevideo: El Arqa (pag. 28 y 29)

RESSANO GARCIA LAMAS, José Manuel (1993). Morfología e desenho de Cidade. Lisboa, Fundaçao Calouste Gulbenkian. Segunda edición 1993. ISBN 972-31-0606-X

SANCHEZ ROSSEL, Astrid (2007). Orientaciones estratégicas para el plan director del Sistema Patrimonial Industrial Anglo. Montevideo: Intendencia Municipal de Río Negro. 